\title{
CHEBYSHEV POLYNOMIALS, ZOLOTAREV POLYNOMIALS AND PLANE TREES
}

\author{
YURY KOCHETKOV
}

\begin{abstract}
A polynomial with exactly two critical values is called a generalized Chebyshev polynomial. A polynomial with exactly three critical values is called a Zolotarev polynomial. Two Chebyshev polynomials $f$ and $g$ are called Z-homotopic, if there exists a family $p_{\alpha}, \alpha \in[0,1]$, where $p_{0}=f, p_{1}=g$ and $p_{\alpha}$ is a Zolotarev polynomial, if $\alpha \in(0,1)$. As each Chebyshev polynomial defines a plane tree (and vice versa), Z-homotopy can be defined for plane trees. In this work we prove some necessary geometric conditions for plane trees Z-homotopy, describe Z-homotopy for trees with 5 and 6 edges and study one interesting example in the class of trees with 7 edges.
\end{abstract}

\section{INTRODUCTION}

1.1. Generalized Chebyshev polynomials. Polynomial $p(z) \in \mathbb{C}[z]$ is called a generalized Chebyshev polynomial if it has exactly two finite critical values $-\alpha$ and $\beta$ (in what follows we will call such polynomial simply a Chebyshev polynomial). If $p(z)$ is a Chebyshev polynomial, then the set $p^{-1}[\alpha, \beta]$ is a plane connected tree $T_{p}$ (see, [1, for example). Inverse images of points $\alpha$ and $\beta$ are vertices of tree $T_{p}$ and the degree of a vertex equals to the multiplicity of the corresponding critical point (a vertex of degree 1 is a simple root of polynomial $p(z)-\alpha$ or $p(z)-\beta$ ). Also for each plane tree $T$ there exists a Chebyshev polynomial $p(z)$, defined up to linear change of variable $z$ and variable $u=p(z)$, such that trees $p^{-1}[\alpha, \beta]$ and $T$ are isotopic. Such polynomial $p(z)$ will be called a polynomial that defines the tree $T$.

Vertices of a plane tree $T$ can be painted in two colors - black and white so, that colors of any two adjacent vertices are different. Such painting will be called a binary structure of $T$. Obviously, vertices of one color are inverse images of $\alpha$ and vertices of another another color - of $\beta$.

The type (or passport) of plane tree with binary structure is two sequences of multiplicities of white vertices and black vertices, respectively, in nonincreasing order. Thus the type of the tree

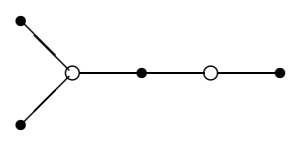

is $\langle 3,2 \mid 2,1,1,1\rangle$.

Remark 1. Often it is assumed that numbers $\alpha$ and $\beta$ are 0 and 1 .

Date: 19.12 .2012 . 
1.2. Zolotarev polynomials. A polynomial $p \in \mathbb{C}[z]$ is called a Zolotarev polynomial if it has exactly three finite critical values. If $p$ is a Zolotarev polynomial, $\operatorname{deg}(p)=n, \alpha, \beta$ and $\gamma$ its critical values and $C$ is a simple arc $C \subset \mathbb{C}$, that connects points $\alpha, \beta$ and $\gamma$, then $p^{-1}(C)$ is a connected plane tree with $2 n$ edges. Here points from the set $p^{-1}\{\alpha, \beta, \gamma\}$ are vertices of this tree and degree of a vertex $v, p(v)=\alpha$, equals to multiplicity of critical point $v$, if $\alpha$ is an endpoint of $C$, or to double multiplicity, if $\alpha$ is an interior point. Vertices of the tree $p^{-1}(C)$ can be painted in three colors: white, black and grey, where white vertices are inverse images of the interior (with respect to $\operatorname{arc} C$ ) critical value. One vertex of each edge is white and other - black or grey.

Remark 2. Arcs $C_{1}$ and $C_{2}$, that connect points $\alpha, \beta$ and $\gamma$, can be isotopically nonequivalent

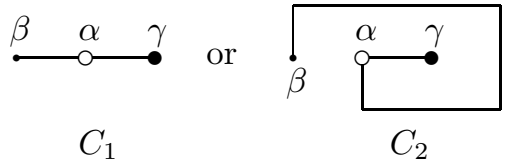

for example. In this case trees $p^{-1}\left(C_{1}\right)$ and $p^{-1}\left(C_{2}\right)$ also can be isotopically nonequivalent.

The passport of Zolotarev polynomial is three sequences of multiplicities of its critical points that correspond to the first, the second and the third critical value, respectively. Multiplicity sequences will be written in the nonincreasing order $\left\langle k_{1}, k_{2}, \ldots\left|l_{1}, l_{2}, \ldots\right| m_{1}, m_{2}, \ldots\right\rangle$. Critical points of polynomial $p=$ $x^{2}(x-1)^{2}(3 x-1)$, for example, are $0,1,2 / 3$ and $1 / 5$ with values $0,0,4 / 81$ and $-32 / 3125$, respectively. So $\langle 2,2|2| 2\rangle$ is the passport of $p$.

\section{Z-номотоРу}

Definition 1. Two trees $T_{1}$ and $T_{2}$ will be called Z-homotopic if there exists a continuous family $p_{\lambda} \in \mathbb{C}[z], \lambda \in[0,1]$, such that

- all polynomials $p_{\lambda}$ has the same degree;

- polynomial $p_{0}$ is a Chebyshev polynomial and defines the tree $T_{1}$;

- polynomial $p_{1}$ is a Chebyshev polynomial and defines the tree $T_{2}$;

- polynomials $p_{\lambda}, \lambda \neq 0,1$, are Zolotarev polynomials, but not Chebyshev polynomials.

Example 1. Let us study the Z-homotopy problem on the set of 5-edge trees. There are five of them:

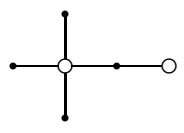

$T_{1}$

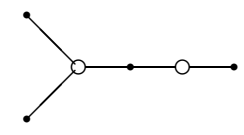

$T_{2}$

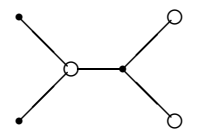

$T_{3}$

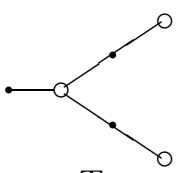

$T_{4}$

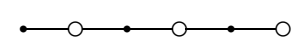

$T_{5}$ 
Let $p=\int x^{2}(x-1)(x-a) d x$. Critical points of $p$ are 0,1 and $a$ and $0,5 a-3$ and $a^{4}(5-3 a)$ are corresponding critical values. If

- $a=0$, then $p$ is a Chebyshev polynomial that defines the tree $T_{1}$;

- $a=1$, then $p$ is a Chebyshev polynomial that defines the tree $T_{3}$;

- $a=3 / 5$, then $p(1)=0$ and $p$ is a Chebyshev polynomial that defines the tree $T_{2}$;

- $a=5 / 3$, then $p(a)=0$ and $p$ is a Chebyshev polynomial that defines the tree $T_{2}$;

- $a=(-2 \pm \sqrt{5} i) / 3$, then $p(a)=p(1)$ and $p$ is a Chebyshev polynomial that defines the tree $T_{4}$.

For all other values of parameter $a$ the polynomial $p$ is Zolotarev polynomial. Thus deformations of parameter $a$ allows one to realize pairwise Z-homotopies between trees $T_{1}, T_{2}, T_{3}$ and $T_{4}$. For example the following deformation of tree corresponds to the increase of parameter $a$ from 0 to $3 / 5$ ( $\operatorname{arc} C$ in this case is the segment, that connects critical values $5 a-3$ and $\left.a^{3}(5-3 a)\right)$ :

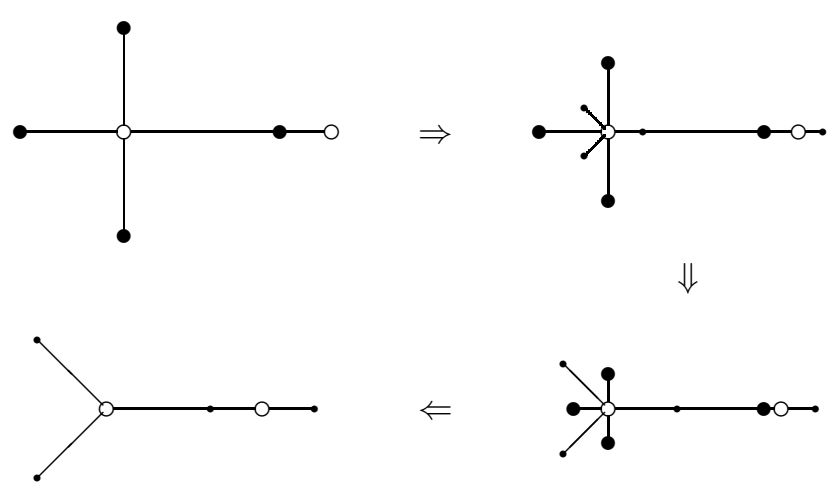

Trees $T_{1}, T_{2}$ and $T_{4}$ are Z-homotopic to tree $T_{5}$. Indeed, let us consider the polynomial $p(x)=\int x(x-1)(x-a)(x-b) d x$. If $p(a)=p(0), a \neq 2$, then this polynomial is a Zolotarev polynomial (here $\left.b=\left(3 a^{2}-5 a\right) /(5 a-10)\right)$. However, for some values of parameter $a$ polynomial $p$ degenerates into Chebyshev polynomial. Indeed,

(1) if $a=0$, then $b=0$, and we have a Chebyshev polynomial, that defines the tree $T_{1}$;

(2) if $a=1$, then $b=2 / 5$ and $p(1)=0$, and we have a Chebyshev polynomial, that defines the tree $T_{2}$;

(3) if $a=5 / 3$, then $b=0$, and we have a Chebyshev polynomial, that defines the tree $T_{2}$;

(4) if $a= \pm \sqrt{5}$, then $b=1 \pm \sqrt{5}$ and $p(1)=p(b)$, and we have a Chebyshev polynomial, that defines the tree $T_{5}$;

(5) if $a=(5 \pm \sqrt{5}) / 4$, then $b=-(1 \pm \sqrt{5}) / 4$ and $p(1)=p(b)$, and we have a Chebyshev polynomial, that defines the tree $T_{5}$;

(6) if $a=(5 \pm \sqrt{5} i) / 3$, then $b=1$, and we have a Chebyshev polynomial, that defines the tree $T_{4}$.

Thus, a deformation of parameter $a$ allows us to construct a Z-homotopy between trees $T_{1}$ and $T_{5}, T_{2}$ and $T_{5}, T_{4}$ and $T_{5}$. 
Trees $T_{3}$ and $T_{5}$ are not Z-homotopic. This statement will be proved in the next section. Also it is a consequence of results in section "Theorems".

\section{Geometry of space of Zolotarev polynomials of Degree 5}

Let $q=x^{4}+a x^{2}+b x+c$ and $p=\int q d x$. The polynomial $p$ is a Zolotarev polynomial if among numbers $p\left(x_{1}\right), p\left(x_{2}\right), p\left(x_{3}\right), p\left(x_{4}\right)$, where $x_{1}, x_{2}, x_{3}, x_{4}$ are roots of $q$, there are only three different. In this case the polynomial $s(y)=\left(y-p\left(x_{1}\right)\right)(y-$ $\left.p\left(x_{2}\right)\right)\left(y-p\left(x_{3}\right)\right)\left(y-p\left(x_{4}\right)\right)$ has a multiple root., i.e. its discriminant is zero. This discriminant is reducible:

$$
\begin{gathered}
\left(1280 a^{6}-32256 a^{4} c+9504 a^{3} b^{2}+269568 a^{2} c^{2}-69984 a b^{2} c-19683 b^{4}-746496 c^{3}\right) \times \\
\left(16 a^{4} c-4 a^{3} b^{2}-128 a^{2} c^{2}+144 a b^{2} c-27 b^{4}+256 c^{3}\right)=0 .
\end{gathered}
$$

We see that the variety of Zolotarev polynomials of degree 5 is reducible and has two components $C_{1}$ and $C_{2}$. The second factor, that defines the component $C_{2}$, is simply the discriminant of polynomial $q$.

Intersection $C_{1} \cap C_{2}$ is the union of 3 components.

- Polynomials that belong to the first component are Chebyshev polynomials that define the tree $T_{4}$.

- Polynomials that belong to the second component are Chebyshev polynomials that define the tree $T_{2}$.

- Polynomials that belong to the third component are Chebyshev polynomials that define the tree $T_{1}$.

A Chebyshev polynomial $p_{0}$ that defines $T_{5}$ belongs only to the first component $C_{1}$ and a Chebyshev polynomial $p_{1}$ that defines $T_{3}$ belongs only to the second component $C_{2}$. Thus a family of Zolotarev polynomials which connect $p_{0}$ and $p_{1}$ must also contain one of Chebychev polynomials in $C_{1} \cap C_{2}$. But then this family is not a Z-homotopy.

\section{THEOREMS}

In this section we will prove a necessary condition for Z-homotopy existence (i.e. a sufficient condition for its absence).

Lemma 1. Let $p_{\lambda}, 0<\lambda<1$ be a continuous family of Zolotarev polynomials of degree $n$. Then passports of all these polynomials are the same.

Proof. Let $a_{\lambda}, b_{\lambda} c_{\lambda}$ be critical values of polynomial $p_{\lambda}$. They are continuous functions of parameter $\lambda$. A change of passport during increase or decrease of parameter $\lambda$ can occur only in the case of collision of roots of polynomial $p_{\lambda}-a_{\lambda}$ (or $p_{\lambda}-b_{\lambda}$, or $p_{\lambda}-c_{\lambda}$ ): two roots $x_{\lambda}^{\prime}$ and $x_{\lambda}^{\prime \prime}$ of polynomial $p_{\lambda}-a_{\lambda}$ of multiplicities $k^{\prime}$ and $k^{\prime \prime}$, respectively, approach to each other, when $\lambda \rightarrow \mu$, and generate a root $x_{\mu}$ of polynomial $p_{\mu}-a_{\mu}$ of multiplicity $k^{\prime}+k^{\prime \prime}-1$.

Let the passport of $p_{\lambda}$ be $\left\langle k_{1}, \ldots, k_{r}\left|l_{1}, \ldots, l_{s}\right| m_{1}, \ldots, m_{t}\right\rangle$. Then

$$
\sum_{i=1}^{r} k_{i}=n, \quad \sum_{i=1}^{s} l_{i}=n, \quad \sum_{i=1}^{t} m_{i}=n
$$

and

$$
\sum_{i=1}^{r}\left(k_{i}-1\right)+\sum_{i=1}^{s}\left(l_{i}-1\right)+\sum_{i=1}^{t}\left(m_{i}-1\right)=n-1 .
$$


Hence, $r+s+t=2 n+1$. But the collision of roots diminishes the number $r$ and violates the above equality.

Remark 3. We see, that it is more correct to speak not about Z-homotopy, but about Z-homotopy in the class of Zolotarev polynomials with a given passport. Thus, trees $T_{1}, T_{2}, T_{3}$ and $T_{4}$ with 5 edges are pairwise Z-homotopic in the class of Zolotarev polynomials with the passport $\langle 3|2| 2\rangle$ and trees $T_{1}$ and $T_{5}, T_{2}$ and $T_{5}$, $T_{4}$ and $T_{5}$ are Z-homotopic in the class of Zolotarev polynomials with the passport $\langle 2,2|2| 2\rangle$.

Lemma 2. Let $p_{\lambda}, 0 \leqslant \lambda<1$, be a continuous family of polynomials of degree $n$, where $p_{0}$ is a Chebyshev polynomial and $p_{\lambda}, \lambda>0$, are Zolotarev polynomials (but not Chebyshev polynomials). Let us assume that a critical point a of polynomial $p_{0}$ of multiplicity $k$ generates $m, m>1$, critical points $a_{1}(\lambda), \ldots, a_{m}(\lambda)$ in the family $p_{\lambda}$ with multiplicities $k_{1}, \ldots, k_{m}$. Then numbers $p_{\lambda}\left(a_{1}(\lambda)\right), \ldots, p_{\lambda}\left(a_{m}(\lambda)\right)$ cannot all be equal.

Proof. Let us assume that the opposite is true:

$$
p_{\lambda}\left(a_{1}(\lambda)\right)=\ldots=p_{\lambda}\left(a_{m}(\lambda)\right)=\alpha(\lambda) .
$$

Let $\lambda \rightarrow 0$. Then

$$
a_{i}(\lambda) \rightarrow a, i=1, \ldots, m, \text { and } \alpha(\lambda) \rightarrow \alpha=p_{0}(a) .
$$

But $k-1=\left(k_{1}-1\right)+\ldots+\left(k_{m}-1\right)$, so $a$ is a root of polynomial $p_{0}-\alpha$ of multiplicity $k+m-1$. We have a contradiction.

Definition 2. A tree is called a chain, if valences of all its vertices are $\leqslant 2$.

Theorem 1. If a tree $T$ has a white vertex a of degree $\geqslant 3$ and a black vertex $b$ of degree $\geqslant 3$, then it cannot be Z-homotopic to a chain.

Proof. Let us assume that the opposite is true. Then there exist a Z-homotopy connecting a Chebyshev polynomial $p_{0}$, that defines $T$, with a Chebyshev polynomial $p_{1}$, that defines the chain. It means that critical points $a$ and $b$ in the family $p_{\lambda}$ generated critical points $a_{1}, \ldots, a_{m}$ and $b_{1}, \ldots, b_{n}$, respectively, all of them of multiplicity 2. Let $p_{0}(a)=\alpha$ and $p_{0}(b)=\beta$. If parameter $\lambda$ is small, then values $p_{\lambda}\left(a_{1}\right), \ldots, p_{\lambda}\left(a_{m}\right)$ are close to $\alpha$ and among them are at least two different. Analogously, values $p_{\lambda}\left(b_{1}\right), \ldots, p_{\lambda}\left(b_{n}\right)$ are close to $\beta$ and among them are at least two different. But then a polynomials $p_{\lambda}, \lambda \ll 1$, has at least 4 critical values. We have a contradiction.

Corollary 1. Trees $T_{3}$ and $T_{5}$ cannot be Z-homotopic.

\section{Trees With SIX EDGes}

Below are all plane 6-edge trees up to mirror symmetry (the designation of symmetrical tree is in brackets):

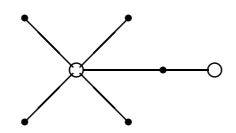

$T_{1}$

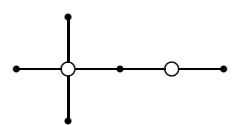

$T_{2}$

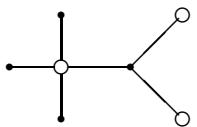

$T_{3}$

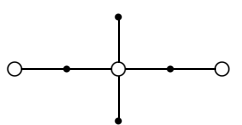

$T_{4}$ 


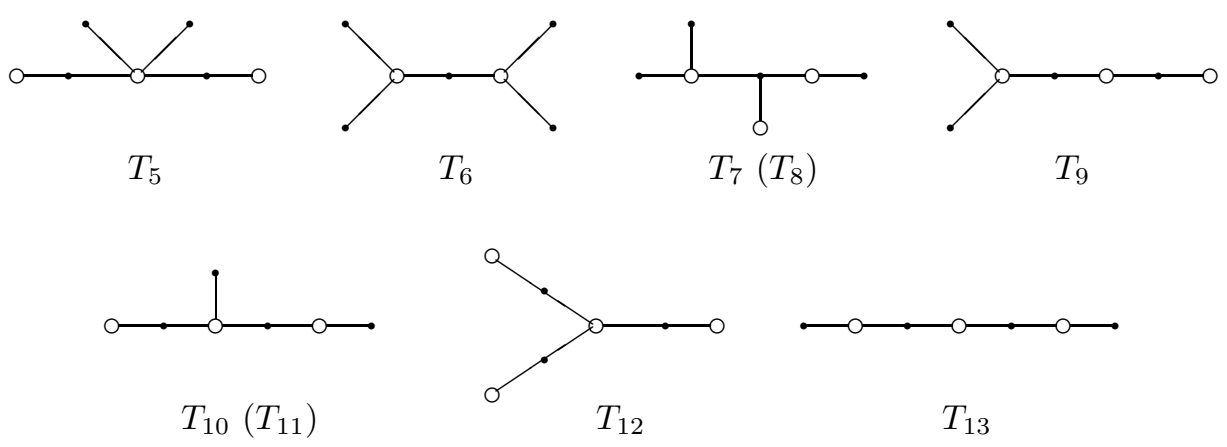

By Theorem 1 from the previous section, trees $T_{3}$ and $T_{13}, T_{7}$ and $T_{13}, T_{8}$ and $T_{13}$ are not Z-homotopic. However, there is one more non-homotopic pair.

Proposition 1. Trees $T_{6}$ and $T_{12}$ are not $Z$-homotopic.

Proof. Let the opposite be true and let $a$ and $b$ be white vertices of degree 3 of the tree $T_{6}$.

The first case. Let polynomials $p_{\lambda}$ have a critical point $a_{\lambda}$ of multiplicity 3 , all other critical points are of multiplicity 2 . Thus the vertex $b$ generates two critical points $b_{1}$ and $b_{2}$ of multiplicity $2, p_{\lambda}\left(b_{1}\right) \neq p_{\lambda}\left(b_{2}\right)$ and value $p_{\lambda}(a)$ coincides with value $p_{\lambda}\left(b_{1}\right)$ or with values $p_{\lambda}\left(b_{2}\right)$. But then tree $T_{12}$ has a white vertex of degree 2 except white vertex of degree 3 .

The second case. Polynomials $p_{\lambda}$ have critical points only of multiplicity 2 . Thus vertices $a$ and $b$ generate critical points $a_{1}, a_{2}$ and $b_{1}, b_{2}$, respectively. Moreover, $p_{\lambda}\left(a_{1}\right)=p_{\lambda}\left(b_{1}\right), p_{\lambda}\left(a_{2}\right)=p_{\lambda}\left(b_{2}\right)$ and $p_{\lambda}\left(b_{1}\right) \neq p_{\lambda}\left(b_{2}\right)$. Let the fifth critical point be $c=c_{\lambda}$. The vertex of $T_{12}$ of degree 3 cannot be generated by junction of points $a_{1}$ and $b_{1}$ (or $a_{2}$ and $b_{2}$ ), because otherwise during the change of parameter $\lambda$ from 1 to 0 the vertex of degree 3 of $T_{12}$ generates two critical points with same values. Also, this vertex cannot be generated by junction of points $c$ and $a_{1}$ (for example), because then $T_{12}$ has a vertex of degree 3 and a vertex of degree 2 of the same color.

All other pairs of trees are Z-homotopic. The construction of corresponding Zhomotopy usually is quite straightforward. Let us describe some interesting cases.

- Tree $T_{4}$ and tree $T_{12}$. Let degree 2 vertices of $T_{4}$ be in points \pm 1 , its degree 4 vertex - in origin, degree 3 vertex of $T_{12}$ - in origin and its degree 2 vertices - in cubic roots of 1 .

Let us consider the polynomial $p=\int x^{2}(x-1)(x-a)(x-b) d x$ with condition $p(a)=p(b)$. Then $p$ is a Zolotarev polynomial with passport $\langle 3|2,2| 2\rangle$. If $a=0$ and $b=-1$, then $p$ degenerates into Chebyshev polynomial that corresponds to the tree $T_{4}$. The change of parameter $a$ from 0 to $-i$, to $2-i$, to 2 , to $2+\sqrt{3} i / 2$ and to $(-1+\sqrt{3} i) / 2$ induces the change of the parameter $b$ from -1 to $(-1-\sqrt{3} i) / 2$.

- Tree $T_{10}$ and tree $T_{13}$. Let degree 3 vertex of $T_{10}$ be in origin, its degree 2 vertices - in points $1, a_{1} \approx 1.57-0.03 i$ and $b_{1} \approx-0.57+0.58 i$, degree 2 vertices of $T_{13}$ - in points $0, \pm 1 \pm \sqrt{3}$.

Let us consider the polynomial $p=\int x(x-1)(x-a)(x-b)(x-c) d x$ with conditions $p(a)=0$ and $p(b)=p(c)$. Then $p$ is a Zolotarev polynomial 
with passport $\langle 2,2|2,2| 2\rangle$. If $a=a_{1}, b=b_{1}$ and $c=0$, then $p$ degenerates into Chebyshev polynomial that corresponds to the tree $T_{10}$. The change of parameter $b$ from $b_{1}$ to -1 induces the change of the parameter $a$ from $a_{1}$ to $\sqrt{3}$ and the change of the parameter $c$ from 0 to $-\sqrt{3}$ (here $c$ moves along the arc in the lower half plane).

- Tree $T_{12}$ and tree $T_{13}$. Let degree 3 vertex of $T_{12}$ be in the point $i / \sqrt{3}$, its degree 2 vertices - in points \pm 1 and $\sqrt{3} i$, degree 2 vertices of $T_{13}$ - in points $0, \pm 1 \pm 1 / \sqrt{3}$.

Let us consider the polynomial $p=\int\left(x^{2}-1\right)(x-a)(x-b)(x-c) d x$ with conditions $p(-1)=p(1)=p(c)$. Then $p$ is a Zolotarev polynomial with passport $\langle 2,2,2|2| 2\rangle$. If $a=b=i / \sqrt{3}$ and $c=\sqrt{3} i$, then $p$ degenerates into Chebyshev polynomial that corresponds to the tree $T_{12}$. The change of parameter $a$ from $i / \sqrt{3}$ to $1 / \sqrt{3}$ induces the change of the parameter $b$ from $i / \sqrt{3}$ to $-1 / \sqrt{3}$ and the change of the parameter $c$ from $\sqrt{3} i$ to 0 .

\section{TREes With SEVEN EDGES}

Zolotarev polynomials of degree 7 with passport $\langle 2,2|2,2| 2,2\rangle$ give a nontrivial example of absence of Z-homotopy (nontrivial in the sense, that this absence cannot be explained by Lemma 2 or Theorem 1 ). Without loss of generality we can assume, that the first critical value is 0 and that corresponding critical points are 0 and 1 . Then such polynomial is of the form

$$
p(x)=\int x(x-1)(x-a)(x-b)(x-c)(x-d) d x,
$$

where

$$
p(1)=0, \quad p(a)=p(b), \quad p(c)=p(d) .
$$

Algebraic variety $C$ in 4 -dimensional space with coordinates $a, b, c, d$, defined by these conditions, is reducible: it is the union of two components $C=C_{1} \cup C_{2}$ of degrees 8 and 16, respectively. Trees (up to mirror symmetry), that correspond to Zolotarev polynomials from the first component, can be seen in the picture below:

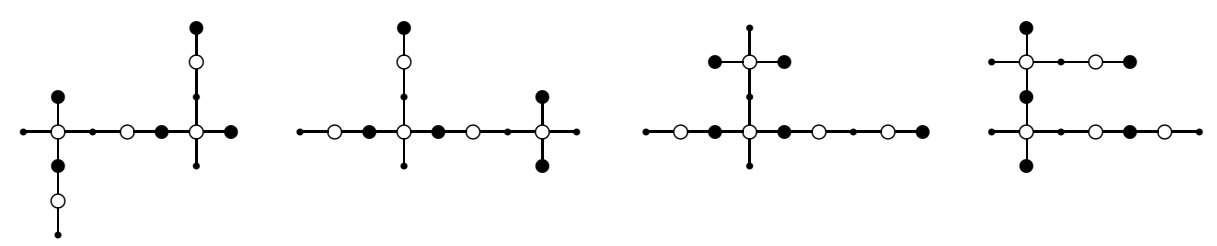

The order of monodromy group of Zolotarev polynomials from $C_{1}$ is 168 .

Trees (up to mirror symmetry), that correspond to Zolotarev polynomials from the second component, can be seen in the picture below:

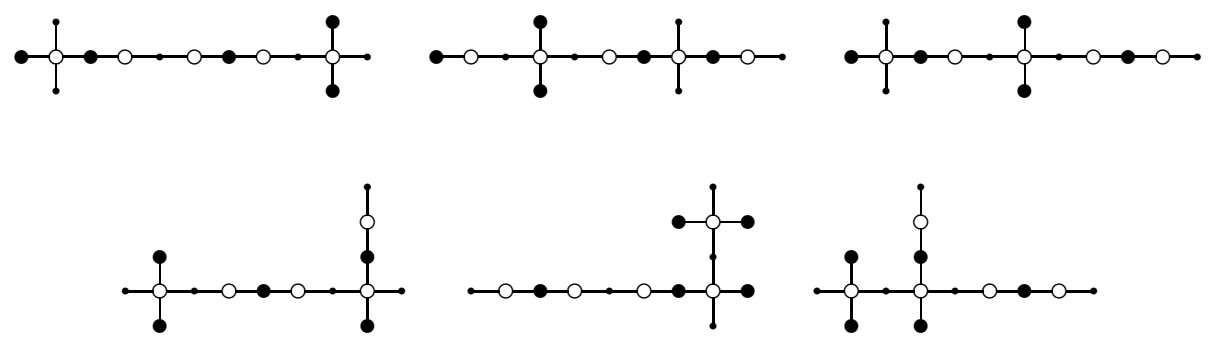



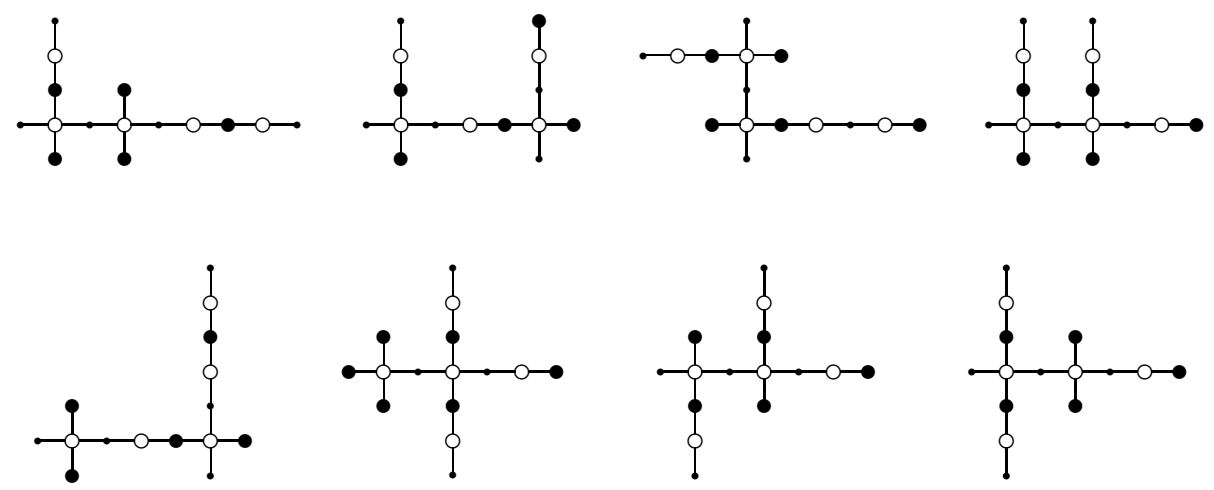

The order of monodromy group of Zolotarev polynomials from $C_{2}$ is 2520 .

The intersection $C_{1} \cap C_{2}$ consists of Chebyshev polynomials that correspond to trees

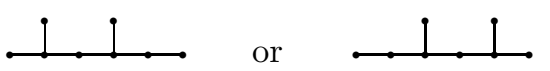

However, the component $C_{1}$ contains Chebyshev polynomials that correspond to trees

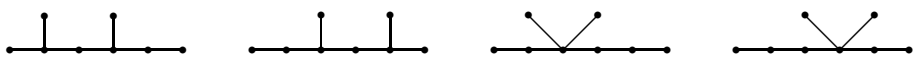

and the component $C_{2}$ contains Chebyshev polynomials, that correspond to trees

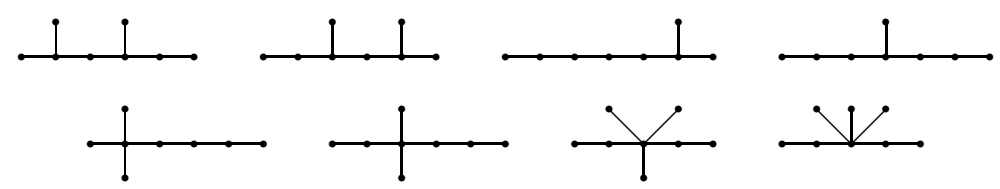

Thus we see that trees

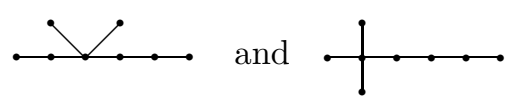

for example, are not Z-homotopic in the class of Zolotarev polynomials with the passport $\langle 2,2|2,2| 2,2\rangle$ (although they are Z-homotopic in the class with the passport $\langle 4|2| 2\rangle)$.

\section{REFERENCES}

[1] Lando S., Zvonkin A. Graphs on surfaces and their applications, Springer, 2004.

E-mail address: yuyk@prov.ru 